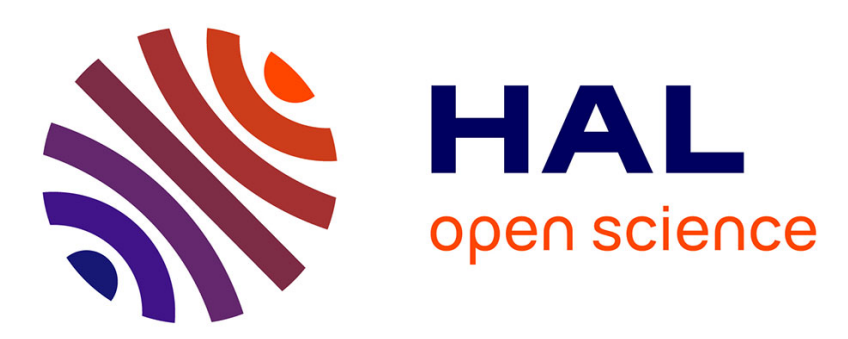

\title{
Análisis de la interacción social en contextos de movilidad transnacional y de superdiversidad
}

\author{
Santiago Sánchez Moreano
}

\section{To cite this version:}

Santiago Sánchez Moreano. Análisis de la interacción social en contextos de movilidad transnacional y de superdiversidad. Iberoromania: Revista dedicada a las lenguas y literaturas iberorrománicas de Europa y América, inPress. hal-02495793

\section{HAL Id: hal-02495793 \\ https://hal.science/hal-02495793}

Submitted on 2 Mar 2020

HAL is a multi-disciplinary open access archive for the deposit and dissemination of scientific research documents, whether they are published or not. The documents may come from teaching and research institutions in France or abroad, or from public or private research centers.
L'archive ouverte pluridisciplinaire HAL, est destinée au dépôt et à la diffusion de documents scientifiques de niveau recherche, publiés ou non, émanant des établissements d'enseignement et de recherche français ou étrangers, des laboratoires publics ou privés. 
À paraître dans Contacto y migración. Desafíos metodológicos en la sociolingüística hispánica actual. Special issue, Iberoromania, De Gruyter, 2020.

\title{
Análisis de la interacción social en contextos de movilidad transnacional y de superdiversidad
}

\author{
Santiago Sánchez Moreano
}

\section{Resumen}

En contextos de superdiversidad y movilidad transnacional, al disponer de repertorios plurilingües, los hablantes tienen la posibilidad de utilizar formas lingüísticas particulares indexicales y socioculturalmente asociadas a las diferentes lenguas, variedades diatópicas, estilísticas o registros (Agha 2005) a los que han estado expuestos a lo largo de sus trayectorias de vida (Busch 2012). Dichas formas lingüísticas, a veces híbridas e innovadoras, pueden ser utilizadas con fines pragmáticos como el de adoptar una postura social particular. Basado en ejemplos conversacionales de Cayena (Guayana Francesa) y Cali (Colombia), este artículo sugiere combinar metodológicamente el análisis conversacional y de la interacción con la observación etnográfica y la teoría de stance (Du Bois 2007) para hacer más fino y productivo el análisis de las prácticas discursivas de los hablantes en su conexión con el mundo social.

\section{Palabras clave}

stance, superdiversidad, movilidad transnacional, análisis del discurso, sociolingüística de la interacción, enfoques etnográficos

\section{Introducción}

El estudio de las movilidades transnacionales (Vertovec 2009) constituye un campo de investigación que se ha venido nutriendo de las reflexiones hechas en disciplinas como la sociología, la antropología social y lingüística, la economía, las ciencias políticas, y más recientemente, la sociolingüística. Gracias a estos estudios se ha pasado, en los últimos años, de tener una visión lineal de los flujos migratorios basada únicamente en la variable etnicidad (Vertovec 2007a: 1025) a una visión más dinámica de la movilidad basada en numerosas y diversas variables. Algunas de estas variables son, por ejemplo, las trayectorias múltiples de los actores sociales, las relaciones transnacionales y transterritoriales que ellos construyen permanentemente, el contacto de grupos con distintos estatus migratorios, los orígenes étnicos diversos, la diversidad lingüística y sociocultural de las sociedades contemporáneas, el estatus socioeconómico de las personas, las desigualdades en el acceso a los derechos ciudadanos como la salud y la educación, las relaciones asimétricas entre grupos mayoritarios y minoritarios, el peso de ideologías dominantes, etc.

La interacción de estas y otras variables es lo que Vertovec (2007b) denomina superdiversidad. El término fue inicialmente utilizado para tratar de comprender el carácter disperso del transnacionalismo en los estudios migratorios en Europa, pero ha sido utilizado para describir la "diversificación de la diversidad" en otros contextos urbanos en el mundo (Meissner \& Vertovec 2015: 541). La superdiversidad describe situaciones en las que el flujo de personas de diferentes orígenes, que mantienen conexiones transnacionales (pero también transterritoriales), es cada vez más complejo y disperso (Spotti \& Blommaert 2017: 169).

Por su parte, la sociolinguística crítica de corte posestructuralista retoma los conceptos de transnacionalismo y superdiversidad en el análisis de las prácticas comunicativas asociadas a la des-territorialización de las sociedades contemporáneas (García, Flores \& Spotti 2016: 9). En efecto, en contextos de superdiversidad, la movilidad transnacional juega un papel importante en la modificación y complejización de los repertorios lingüísticos de los actores 
À paraître dans Contacto y migración. Desafíos metodológicos en la sociolingüística hispánica actual. Special issue, Iberoromania, De Gruyter, 2020.

sociales. Mientras que los estudios sobre migración se limitan a la descripción y al análisis de factores como la integración, la asimilación, el racismo, el aprendizaje o el desplazamiento de la lengua, etc., el transnacionalismo y la superdiversidad tienen más en cuenta la agentividad de los actores sociales en la construcción de sus trayectorias de vida y en la modificación de sus repertorios lingüísticos. Así, al disponer de repertorios plurilingües, en constante movilidad, los hablantes tienen la posibilidad de utilizar formas lingüísticas particulares, a veces híbridas e innovadoras, con fines pragmáticos, como el de adoptar una postura social particular con respecto a algo o a alguien en función de los requerimientos del contexto sociocultural y de interacción.

En este artículo, se trata, entonces, de la manera cómo los individuos, en contextos de movilidad transnacional y de superdiversidad, pueden adoptar posturas sociales a través del uso de formas linguíisticas particulares, indexicales y socioculturalmente asociadas a las diferentes lenguas, variedades diatópicas o estilísticas, pero también a registros o voces estereotipadas (Agha 2005), etc. a las que han estado expuestos a lo largo de sus trayectorias de vida y que son constitutivos de sus repertorios lingüísticos (Busch 2012, 2017). Esta perspectiva, centrada más en los hablantes y en sus repertorios linguiísticos, nos permite comprender cómo dichos rasgos son utilizados dinámicamente en la interacción, reconocidos por los hablantes, y utilizados con fines específicos como el de asumir una postura social.

Para comprender esto se propone que el análisis conversacional y de la interacción debe estar acompañado de la observación etnográfica de posturas sociales (stance). A su vez, estas posturas nos pueden ayudar a comprender cómo los hablantes negocian significados sociales e identidades en la interacción en este tipo de contextos. Esta combinación de análisis conversacional y de la interacción, de observación etnográfica, y de la teoría de stance puede contribuir a hacer más fino y productivo el análisis de las prácticas de lenguaje de los hablantes. Además, se propone que el nivel de análisis, en términos lingüísticos, no debe hacerse al nivel del manejo relativo, adecuado y correcto de lenguas como entidades supranacionales, autónomas, y fácilmente identificables; es decir, construcciones socioculturales ideológicamente constituidas. Más bien, el nivel de análisis debe hacerse a nivel de linguistic features (Jørgensen et al. 2011) o de recursos lingüísticos (Blommaert y Backus 2011) particulares que pueden estar asociados socioculturalmente a las construcciones ideológicas que llamamos lenguas. El uso de estas formas particulares se hace en el marco de prácticas de lenguaje heterogéneas (Léglise 2013) que contribuyen a la construcción del mundo social, puesto que hacen parte de él y están determinadas/supeditas a él.

En la sección 1 se discute la necesidad de pasar del estudio de lenguas a la comprensión del uso que hacen los hablantes de recursos lingüísticos asociados a diferentes lenguas. En la sección 2 se discute la importancia de comprender cómo se modifican los repertorios lingüísticos de los actores sociales en situación de movilidad transnacional y gracias a trayectorias de vida diferenciadas. Se ilustrará esto a partir de la situación de movilidad transnacional de peruanos en la Guayana francesa y de quichuas ecuatorianos en la ciudad de Cali, en Colombia. En la sección 3 se resalta la importancia de la observación etnográfica en los análisis conversacionales y de la interacción para comprender el uso concreto que hacen los hablantes de sus recursos lingüísticos. En la sección 4 se propone, a partir de ejemplos de prácticas de lenguaje etnográficamente observadas en Cayena y Cali, que el uso de recursos lingüísticos particulares contribuye a la adopción de posturas sociales por parte de los hablantes en el curso de la interacción y que estas posturas hacen parte de procesos más amplios de construcción de identidades locales y globales. Al final del artículo se presentan algunas conclusiones y aperturas a otros análisis posibles.

\section{Lenguas, recursos y repertorios lingüísticos}


À paraître dans Contacto y migración. Desafíos metodológicos en la sociolingüística hispánica actual. Special issue, Iberoromania, De Gruyter, 2020.

En los últimos años numerosos estudios en antropología lingüística, en sociolinguística crítica y en linguística aplicada han venido trabajando la idea según la cual las lenguas son entidades sociocultural e ideológicamente construidas que pueden ser fácilmente identificadas y nombradas (Léglise 2018; Makoni \& Pennycook 2005; Heller 2007; Otheguy, García \& Reid 2015). Existe en estos trabajos una discusión crítica del concepto de lengua a través de la cual se ha venido des-construyendo la idea de que se trata de entidades autónomas y separables. Se critica también cómo dicho concepto ha sido construido sobre la base de ideologías normativas, políticas y socio-históricas.

Por ejemplo, Makoni y Pennycook (2005: 138) sugieren que la idea de una lengua, en el sentido más literal, es una invención europea y que los europeos, como parte de un proyecto de evangelización colonial, la impusieron en aquellos lugares que ellos colonizaron denominando arbitrariamente, en muchos casos, las diversas formas de hablar de sus habitantes. Paralelamente a este proceso de invención europeo, surgió la idea de que las lenguas son entidades separables, categorizables y denominables que, además, están asociadas al concepto de Estado-Nación. Es así como encontramos las denominaciones español, portugués, inglés, francés o ruso, como las lenguas que se hablan en España, Portugal, Inglaterra, Francia o Rusia, respectivamente. En este sentido, tanto las lenguas como los Estados-Nación son, en últimas, constructos socioculturales y socio-históricos.

Autores como Otheguy, García y Raid (2015) sugieren también que estas así denominadas lenguas (named languages) no pueden ser lingüísticamente definidas. Es decir, que linguísticamente, no existen criterios que permitan definir la denominación de una lengua como español, portugués o francés. La definición lingüística de lo que delimita o distingue una lengua de otra ya era un problema evocado por Romaine (2000 [1994]: 136 citada por Jørgensen et al. 2011: 27) ${ }^{1}$, quien afirmaba, sobre dos lenguas cercanas como el alemán y el holandés, por ejemplo, que no era posible delimitar las fronteras basándose únicamente en criterios lingüísticos. Esto significaría, por el contrario, que las lenguas y variedades de lengua (dialectos, registros o estilos y otras formas de habla) podrían ser definidas básicamente por criterios externos de tipo sociocultural como la afiliación social, política o étnica de sus hablantes, y también por políticas lingüísticas nacionales o regionales impuestas en un determinado territorio (Jørgensen et al. 2011: 28). Todos estos criterios son necesariamente socioculturales e ideológicos y han contribuido a la instauración de fronteras entre las lenguas y entre las disciplinas que se encargan de su estudio.

A su vez, estas invenciones socio-históricas, así como la instauración de fronteras entre lenguas, han tenido efectos reales y materiales en la forma como las lenguas han sido entendidas, en cómo las políticas lingüísticas han sido construidas, en cómo la educación ha sido concebida y, sobre todo, en cómo la gente ha llegado a identificarse con dichas entidades/lenguas (Makoni y Pennycook 2005: 138). Según Léglise (2018), los lingüistas también hemos contribuido a la instauración de fronteras entre las lenguas al identificar en nuestros corpus bilingües y plurilingües, diferentes lenguas aun cuando, en la interacción, los hablantes suelen, en muchos casos, transgredir dichas fronteras.

A pesar de que se ha avanzado considerablemente en las últimas décadas en la des-construcción de la idea de lenguas como entidades discretas e identificables, cuestionando el concepto estructural del lenguaje (Jørgensen et al. 2011), hablar de lo heterogéneo de las prácticas plurilingües de los hablantes sin recurrir artificialmente al hecho de instaurar fronteras, incluso por parte de los lingüistas, sigue siendo una tarea compleja (Léglise 2018: 2).

Asimismo, para Jørgensen et al. (2011: 27) la idea de lenguas como sistemas delimitados parece ser insuficiente para capturar y comprender el uso real del lenguaje que hacen los hablantes en las sociedades superdiversas modernas, y tal vez, también, en todas las sociedades del mundo.

\footnotetext{
${ }^{1}$ Romaine, Suzanne. (2000): Language in Society: An Introduction to Sociolinguistics ( $2^{\mathrm{a}}$ ed.), Oxford/New York: Oxford University Press
} 
À paraître dans Contacto y migración. Desafíos metodológicos en la sociolingüística hispánica actual. Special issue, Iberoromania, De Gruyter, 2020.

Para aprehender de manera más cercana lo que realmente hacen los hablantes en la interacción social, los autores sugieren tener en cuenta, más bien, un nivel de análisis basado en elementos lingüísticos o linguistic features en lugar de un análisis basado en lenguas. Así, se trataría de analizar el uso de elementos lingüísticos que constituyen lo que socioculturalmente se conoce como español, portugués, italiano, o cualquier otra lengua. En este sentido, los hablantes no utilizamos lenguas, sino linguistic features que están a nuestra disposición y que pueden estar asociados a categorías específicas llamadas lenguas (Jørgensen et al., 2011: 28-29). Por su parte, Blommaert y Backus (2011) prefieren el término recursos lingüísticos para referirse a los linguistic features.

Ahora, dichas formas lingüísticas o recursos lingüísticos son por naturaleza heterogéneos, es decir, que pueden estar socioculturalmente asociados a diferentes lenguas, variedades diatópicas, registros, estilos, acentos u otras formas de hablar o de variación linguiística. La práctica social, que consiste en utilizar dinámicamente dichos recursos heterogéneos en función del contexto social en el que está imbricada la interacción, es lo que Léglise (2018) denomina como pratiques langagières hétérogènes. Para Léglise, basándose en los trabajos de Boutet, Fiala y Simoni-Grumbach (1976), la idea de práctica es fundamental para comprender la imbricación del uso del lenguaje que hacen los hablantes en el mundo social. Así, una pratique langagière es fundamentalmente una práctica social puesto que hace parte del conjunto de prácticas sociales y, por tanto, contribuye a la construcción del mundo social, al mismo tiempo que está determinada por él.

Como lo sugiere Léglise (2018), frente a la imposibilidad de traducir el adjetivo langagier a otras lenguas (como el inglés, por ejemplo), en español, podríamos simplemente hablar de prácticas lingüísticas, pero estaríamos contribuyendo a la identificación de diferentes lenguas en un corpus plurilingüe o en una conversación, por ejemplo. Algo similar pasa con el término prácticas multilingües en el sentido que el prefijo multi remite a la adición y manejo de varias lenguas por parte de los hablantes (Léglise 2018; Jørgensen et al 2011).

El término pratiques langagières hétérogènes de Léglise (2013) y el de polylanguaging de Jørgensen et al. (2011) resultan probablemente más adecuados para describir la diversidad de recursos lingüísticos utilizados por los hablantes en función de las necesidades del contexto social de interacción. Se trata entonces de una visión del ejercicio del lenguaje, como actividad social, en el que lo que resulta importante, no son las estructuras de las lenguas, sino los recursos variados que están a disposición de los hablantes y que hacen parte de sus repertorios lingüísticos; y que, a su vez, están asociados a los constructos socioculturales que llamamos lenguas.

\section{Repertorios lingüísticos y trayectorias de vida diferenciadas en Cayena y en Cali}

A partir de los trabajos de J. Gumperz en sociolinguiística de la interacción en los años sesenta, surge la noción de repertorio lingüístico o verbal que define el conjunto de "todas las formas aceptadas de formular mensajes" y que le da al hablante la posibilidad de escoger aquellas que necesita en la comunicación diaria en función de los significados que quiere transmitir (Gumperz 1964: 38). Para Gumperz, en un repertorio plurilingüe las lenguas y dialectos forman un "todo", más allá de distinciones gramaticales. Así, las lenguas y dialectos son variedades constitutivas del mismo repertorio (Gumperz 1964: 140). Sin embargo, esta definición se focaliza principalmente en los espacios de interacción social y deja de lado aspectos como las dimensiones históricas, ideológicas y biográficas que están asociadas a las trayectorias de vida de los hablantes.

En este sentido, los repertorios lingüísticos de los hablantes no solo se constituyen en espacios de interacción social, sino que se constituyen a lo largo de sus diferentes y diversificadas trayectorias de vida. Esto significa que los procesos de adquisición y aprendizaje de los 
À paraître dans Contacto y migración. Desafíos metodológicos en la sociolingüística hispánica actual. Special issue, Iberoromania, De Gruyter, 2020.

diferentes recursos lingüísticos, i.e. linguistic features asociados a diferentes lenguas o formas de hablar que hacen o harán parte de los repertorios lingüísticos de los hablantes, son fundamentalmente biográficos y multimodales. En términos de Busch (2017), los repertorios lingüísticos se constituyen a través del Spracherleben o la experiencia vivida del lenguaje.

En situaciones de superdiversidad (Blommaert \& Rampton 2011; Vertovec 2007b) y de movilidad transnacional (Vertovec 2007a, 2009), los sujetos no solo construyen lazos e interacciones entre dos o más Estados, sino que también, están permanentemente moldeados y constituidos en su pensamiento, forma de hablar y de actuar, en sus sentimientos y deseos, por el poder, muchas veces normativo y restrictivo, de categorías sociales discursivamente establecidas (Busch 2012), lo que necesariamente tiene implicaciones en sus repertorios lingüísticos.

Tal es el caso de los peruanos inmigrantes que viven en la ciudad de Cayena en la Guayana francesa, un departamento francés de ultramar situado en el plateau amazónico y que cuenta con unos 280.000 habitantes. Los peruanos son un grupo minoritario que hace parte del $30 \%$ de la población inmigrante que vive en ese territorio, mayoritariamente compuesta por brasileros ( $10 \%$ de la población inmigrante), pero también por poblaciones asiáticas, antillanas, etc. Por su diversidad linguística y cultural, es un observatorio fascinante para el estudio de prácticas de lenguaje en relación con la migración, la movilidad social y espacial, y los fenómenos de variación y contacto lingüístico (Migge \& Léglise 2013).

Según las encuestas de Léglise, realizadas en los últimos 20 años ${ }^{2}$, en la Guayana francesa hay unas 40 lenguas. El francés es lengua oficial en este departamento, sin embargo, paradójicamente, el 75\% de la población no habla francés antes de ir a la escuela. El $1 \%$ declara tener el español como lengua de primera socialización, el 3\% declara hablar lenguas asiáticas, dentro de las que se encuentran el hmong, el thai y el chino, y el 10\% declara hablar portugués brasilero. En términos de repertorios linguiísticos, además del francés, casi el $20 \%$ declara tener el portugués brasilero dentro de su repertorio. Menos del $1 \%$ declara tener el hmong en sus repertorios lingüísticos y el español, por su parte, es declarado ser hablado por alrededor del $5 \%$ de la población. Dentro de la población de hispanohablantes se encuentran los peruanos, dominicanos, colombianos y, minoritariamente, ecuatorianos y bolivianos. Muchos de ellos trabajan de manera informal en el mercado de Cayena, un espacio de multilingüismo en el que se refleja la extrema diversidad de este territorio de ultramar.

Algo similar sucede con los quichuas ecuatorianos que viven en Cali (Colombia), quienes también se encuentran en un contexto de diversidad y contacto de poblaciones. La ciudad de Cali cuenta con aproximadamente 1.800 .000 habitantes $^{3}$. Al ser el polo económico más importante del suroccidente colombiano, la ciudad recibe grupos indígenas inmigrantes ${ }^{4}$ de los departamentos vecinos del Cauca, Huila, Nariño y Putumayo, Chocó y de la Costa pacífica (afrocolombianos, indígenas, grupos rurales), pero también grupos transnacionales como en el caso de los quichuas ecuatorianos. ${ }^{5}$

Los quichuas ecuatorianos de Cali provienen de dos provincias ecuatorianas (Imbabura y Chimborazo) y mantienen relaciones transnacionales entre estos dos Estados. Muchos trabajan en el centro de Cali como comerciantes de ropa y productos artesanales. Hacen parte del comercio informal que caracteriza el centro, lo que los lleva a estar en contacto permanente con

\footnotetext{
2 www.vjf.cnrs.fr/sedyl/Isabelle_Léglise/guyane/.

${ }^{3}$ https://sitios.dane.gov.co/cnpv/\#!/. Censo 2018.

4 Algunos de estos grupos indígenas son los kofan, los quichuas, los misak o guambianos, los ingas, los nasa, los yanaconas y los pastos. Al igual que los quichuas, estos grupos han sido constreñidos por diferentes factores sociales, económicos y de seguridad a instalarse en Cali en búsqueda de una mejor calidad de vida.

${ }^{5}$ C.f. Sánchez Moreano 2017, 2018, Motta \& Posso 2007 para más detalle sobre los quichuas y sobre los demás grupos minoritarios presentes en la ciudad.
} 
À paraître dans Contacto y migración. Desafíos metodológicos en la sociolingüística hispánica actual. Special issue, Iberoromania, De Gruyter, 2020.

otros comerciantes informales de la ciudad, con otros grupos indígenas urbanos, y con los clientes que vienen a comprar sus productos.

Como es de esperarse, la presencia de los quichuas en Cali tiene consecuencias sociales importantes: el espacio sociolingüístico es permanentemente configurado por las problemáticas sociales colombianas y mundiales (movilidad, violencia, migración, desplazamientos forzados, desplazamientos económicos, etc.) y sobre todo con implicaciones lingüísticas y sociales aún poco descritas. Se observa una interrupción de la transmisión intergeneracional del quichua y el desarrollo de estrategias sociolingüísticas de adaptación y de resistencia.

Tanto los peruanos de Cayena como los quichuas de Cali se encuentran inmersos en situaciones de diversidad lingüística y sociocultural, ciertamente diferentes, pero que responden a lo que se ha descrito para contextos de superdiversidad y transnacionalismo (diversidad de poblaciones, migraciones, movilidad espacial y social, orígenes étnicos, relaciones transnacionales, diferencias culturales, estatus socio-económicos asimétricos, etc.).

En estos contextos de superdiversidad y transnacionalismo, tanto los peruanos de Cayena como los quichuas de Cali negocian y construyen permanentemente sus identidades sociales en la interacción social gracias a sus repertorios lingüísticos que se han complejizado a través de las diferentes trayectorias de vida que han experimentado. En este tipo de contextos, los hablantes viven expuestos a varias lenguas, variedades de lengua, a variedades estilísticas, registros, formas particulares de hablar, repertorios emergentes, etc. Al mismo tiempo, están expuestos a las creencias, valores e ideologías lingüísticas (Kroskrity 2006) que están ligadas a cada una de esas formas de hablar, lo que genera diferentes formas de negociación de posturas sociales. Dicha negociación puede operar, entre otros, a través del uso de sus recursos lingüísticos particulares, pero también de los contenidos que estos vehiculan.

Así, en las dos secciones que siguen veremos, a partir de ejemplos de prácticas de lenguaje recogidas en estos dos contextos, cómo los hablantes utilizan dinámicamente sus recursos linguísticos variados en función de los requerimientos que exige el contexto sociocultural (regímenes de lenguaje según Kroskrity (2006)) y que puede potenciar o no el despliegue de sus recursos lingüísticos heterogéneos. Para comprender esto, se propone que el análisis conversacional debe estar acompañado de la observación etnográfica de posturas sociales (stance), y que esta última es una herramienta analítica pertinente para comprender cómo los hablantes negocian significados sociales e identidades en la interacción.

\section{Análisis conversacional y observación etnográfica de la interacción en contextos de movilidad}

En sus estudios sobre la significación social de la alternancia de códigos, Peter Auer (1995, 2005), distanciándose de los estudios estructuralistas sobre el codeswitching (Myers-Scotton 2001), subraya la necesidad de analizar el uso y los efectos sociales de los diferentes códigos que hacen los hablantes bilingües en la estructura secuencial de la conversación. Para él, el trabajo del lingüista consiste en reconstruir los procesos sociales asociados al bilingüismo de los hablantes (1995: 115). Dentro de estos procesos, se debe tener en cuenta el hecho de que el significado social de una "alternancia de código" en particular (nosotros preferimos hablar del uso de recursos lingüísticos), realizada en los diferentes turnos de habla de la conversación (Sacks, Schegloff \& Jefferson 1974), dependerá esencialmente de su entorno secuencial (Auer 1995: 116). En este sentido, el análisis de los diferentes turnos de habla pone en evidencia no solo la secuencia conversacional y la organización de las intervenciones de los diferentes hablantes en la conversación, sino también el significado social del uso de formas lingüísticas en términos de alineamientos o des-alineamientos conversacionales. Son estos últimos los que tienen un valor social en la conversación. 
À paraître dans Contacto y migración. Desafíos metodológicos en la sociolingüística hispánica actual. Special issue, Iberoromania, De Gruyter, 2020.

Así, Auer propone que un extracto de conversación puede ser codificado en función del uso secuencial de diferentes códigos que hacen los hablantes y que esta codificación puede dar indicios de (des)alineamientos interaccionales. Por ejemplo, una secuencia conversacional puede expresarse de la siguiente manera: A1 A2 A1 A2 B1 B2 en la que A y B corresponden a dos lenguas o códigos diferentes. Los números 1 y 2 corresponden a los participantes de la conversación. La combinación A1 corresponde al turno de habla del hablante 1, mientras que A2 corresponde al turno de habla del hablante 2. Ambos comparten el mismo código: A, por lo menos en los primeros 4 turnos de habla. Luego, a partir del quinto turno de habla, se observa un cambio de código por parte del hablante 1 quien empieza a utilizar B. Este cambio en la secuencia conversacional es lo que puede resultar socialmente significativo para Auer, sobre todo, si dicho cambio tiene un efecto en lo que sigue de la conversación. De hecho, en el siguiente y último turno de habla, el hablante 2 también utiliza B. En otros términos, el hablante 2 se alinea con el hablante 1 al utilizar el mismo código: $\mathrm{B}$.

El modelo de Auer se puede ver ilustrado de la línea 9 en adelante en el siguiente ejemplo (1) del corpus del mercado de Cayena ${ }^{6}$.

(1)

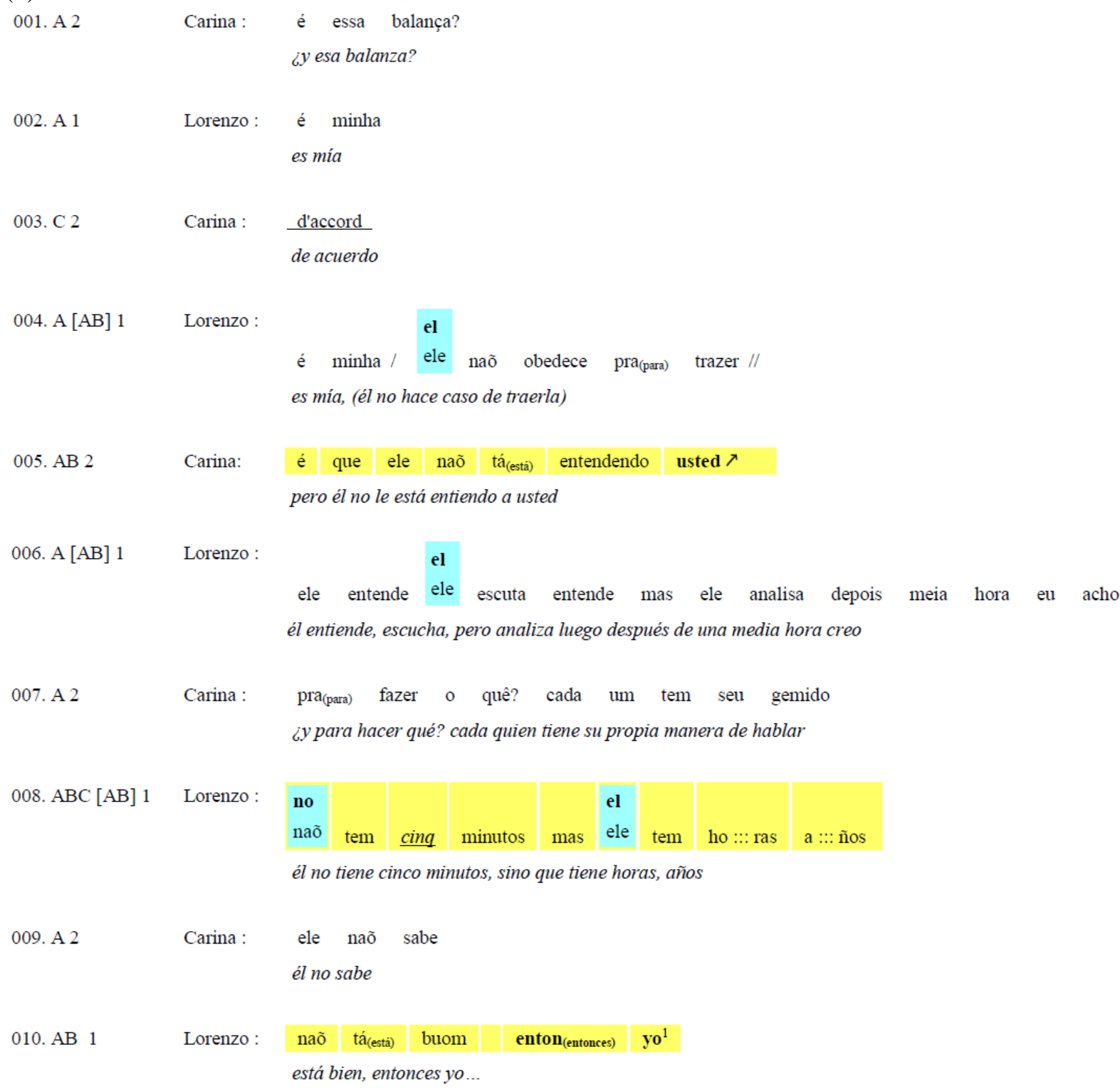

${ }^{6}$ Este corpus de primera mano fue recolectado etnográficamente en 2016 y 2018 en el mercado de Cayena. 
À paraître dans Contacto y migración. Desafíos metodológicos en la sociolingüística hispánica actual. Special issue, Iberoromania, De Gruyter, 2020.

$\begin{array}{llllll}\text { 011. B } 3 & \text { Amigo: } & \text { (singing) } & \text { solamente una vez } \\ \text { 012. B } 1 & \text { Lorenzo: } & \text { (laughter) amé en la vida } \\ \text { 013. B } 3 & \text { Amigo: } & \text { (singing) solamente una vez } & & & \\ \text { 014. B } 1 & \text { Lorenzo: } & & & & \\ & & & & \end{array}$

Se trata de una conversación entre Lorenzo y Carina en la que interviene un amigo de Lorenzo (línea 011). Lorenzo trabaja en el mercado de Cayena como vendedor de frutas y verduras. Vivió varios años en Brasil antes de radicarse en la Guayana francesa, por lo que habla portugués brasilero. En Cayena ha adquirido competencias en otras lenguas como el francés y lenguas asiáticas como el hmong. Por su parte, Carina está radicada desde hace algunos años en la Guayana francesa. Además, del francés y del portugués brasilero, Carina conoce elementos lingüísticos asociados al español que utiliza cuando interactúa con hispanohablantes como se observa al final de la línea 005.

De manera interesante, ambos hablantes comparten elementos lingüísticos del portugués brasilero, del francés y del español, los cuales son utilizados dinámica y fluidamente en la conversación como se observa desde la línea 001 hasta la 010. Esta utilización de elementos lingüísticos variados muestra que los repertorios de los hablantes son heterogéneos, aunque no se evidencia un uso particular que parezca tener un efecto social en la secuencia conversacional, al menos hasta la línea 011 cuando interviene el amigo de Lorenzo, un guayanés que conoce hace varios años. En la secuencia conversacional vemos cómo el amigo interviene en la conversación utilizando B (español) y es seguido por Lorenzo quien termina utilizando también B (línea 012).

Al igual que en el ejemplo de la secuencia de Auer en la que el hablante 2 se alinea con el hablante 1, aquí, Lorenzo parece también alinearse con su amigo en español. Esto se puede interpretar como una negociación en la elección de una lengua o código en la conversación (Auer 1995: 130). Por supuesto, para Auer, la estructura conversacional se define en términos bilingües, esto es, el uso alternante de un código A y un código B, fácilmente distinguibles. La tarea se vuelve más compleja cuando, desde una visión de des-construcción de la idea de lengua o código, describimos prácticas de lenguaje heterogéneas. Estas prácticas son potenciadas por los repertorios linguíísticos heterogéneos multimodales y biográficamente constituidos de los hablantes, y que están asociados a múltiples lenguas, variedades de una misma lengua, dialectos, registros, estilos, etc., reveladores de la superdiversidad de nuestras sociedades contemporáneas como bien lo ilustran las líneas 001 a 010 del ejemplo anterior.

Así, un análisis conversacional nos puede dar indicios de la manera en que los hablantes utilizan dinámicamente sus recursos lingüísticos. Sin embargo, en este estadio, estamos todavía lejos de poder probar que algunas formas lingüísticas particulares pueden estar asociadas socioculturalmente a una forma de hablar en particular (lengua, dialecto, código, registro, estilo, etc.). Estamos también lejos de poder probar que ciertas formas lingüísticas contribuyen a la construcción de identidades locales o nacionales.

Es aquí donde la etnografía adquiere un papel importante en la comprensión de los fenómenos sociolingüísticos e interaccionales como potencialmente creadores de identidad. En este sentido, Bucholtz y Hall (2008: 152) sugieren que, por ejemplo, basarse únicamente en datos empíricos conversacionales no es suficiente y que es importante "fundamentar simultáneamente el estudio de la identidad en datos empíricos interaccionales y contextualizar su estudio a través de la etnografía”. 
À paraître dans Contacto y migración. Desafíos metodológicos en la sociolingüística hispánica actual. Special issue, Iberoromania, De Gruyter, 2020.

La idea de adoptar métodos etnográficos en el estudio de la significación social del lenguaje, que no es novedosa (ver por ejemplo, Hymes 1974), es importante en el sentido en que el etnógrafo no solo tiene que observar la comunicación desde el punto de vista y desde los intereses de la comunidad en la que trabaja, sino que tiene que ver a los miembros de la comunidad como fuentes de conocimiento y entendimiento compartidos (Hymes 1974: 8).

Estos conocimientos compartidos se entienden como lo que socioculturalmente identifica y relaciona a los hablantes al interior de un grupo social en particular, con sus especificidades e identidades particulares, y que se construye en el seno de actos socioculturales y discursivos cotidianos, en la interacción. Estudiar atentamente las especificidades socioculturales de la vida diaria en el momento en el que ocurren y a medida que se reproducen en el tiempo es una tarea necesaria para descubrir la especificidad y el significado social de las prácticas y categorías identitarias locales, así como las maneras en la que estas se construyen en el transcurso de acciones sociales e interacciones repetidas (Bucholtz \& Hall 2008: 152).

La observación etnográfica nos permite afirmar que lo que hacen Lorenzo y Carina en las primeras diez líneas del ejemplo anterior, es decir la utilización de recursos lingüísticos asociados al portugués brasilero, al español y al francés, es una práctica social recurrente entre brasileros y peruanos. En dichas prácticas no se negocian necesariamente una afiliación a un Estado o a otro, sino que se expresa lo heterogéneas y diversificadas que son las trayectorias transnacionales de los que antes se consideraban, en los estudios sobre migraciones, como inmigrantes de un origen étnico determinado.

Según Bucholtz y Hall (2008), basarse en el estudio de las prácticas locales de los hablantes a través de la etnografía puede permitir al investigador comprender cómo los procesos sociohistóricos y sociopolíticos más amplios, asociados a diferentes ideologías lingüísticas, llegan a impactar y a ser implementados por los actores sociales en los procesos de construcción identitaria. Pero también nos puede ayudar a comprender cómo se construyen nuevas identidades a la vez heterogéneas y transnacionales como la de los peruanos y brasileros en Cayena. Para ellas, el análisis de la conversación y otras formas de análisis del discurso son a menudo más productivos cuando están combinados con métodos etnográficos que permiten a los investigadores identificar el sentido de elementos particulares de la interacción, así como aproximarse al contexto sociocultural en el que la interacción tiene lugar y al que está contribuyendo a construir.

Pasar rápidamente del uso de formas y estructuras lingüísticas (o recursos) en la conversación a la construcción de categorías identitarias de la interacción, puede afectar la precisión y la pertinencia de los análisis. Es por esto que es necesario comprender primero cómo los hablantes adoptan posturas sociales en la interacción y cómo su repetición en varios contextos se vuelve indicio de procesos de construcción identitaria tanto a nivel local como general.

Aunque son muchos los trabajos que se han dado a la tarea de comprender el lazo que existe entre las formas lingüísticas y el mundo social (Bucholtz 1999; Mendoza-Denton 2014; Meyerhoff 2009; Kiesling 2004), todavía son pocos los que lo hacen a partir de prácticas de lenguaje heterogéneas en situaciones de contacto lingüístico y de poblaciones, transnacionalismo y superdiversidad como las que se observan en la Guayana francesa y/o en Cali, pero también en muchos otros contextos de superdiversidad en el mundo.

\section{Adopción de posturas sociales en la interacción}

Para evitar ir rápidamente del uso de recursos lingüísticos a la construcción de identidad de los hablantes, se necesita analizar las prácticas de lenguaje en un nivel más fino. Se necesita comprender los efectos concretos de la utilización de ciertas formas linguísticas o recursos lingüísticos por parte de los hablantes en la interacción. Se necesita saber qué hacen los hablantes, interaccionalmente, con dichos recursos, y cómo, a través de ellos, asumen diferentes 
À paraître dans Contacto y migración. Desafíos metodológicos en la sociolingüística hispánica actual. Special issue, Iberoromania, De Gruyter, 2020.

posturas epistémicas y afectivas. Para esto, utilizamos el concepto de stance (Du Bois 2007; Jaffe 2009; Kiesling, 2009; Ochs, 1992), una noción inicialmente introducida por Elinor Ochs (1992) para explicar cómo las formas semióticas están relacionadas con las significaciones sociales y, por ende, con el mundo social.

Las posturas sociales o stance pasan por la adopción de posiciones en la interacción. La acción de posicionarse (positioning), para Davies y Harré (1990), se define como la capacidad de los hablantes de definirse a sí mismos en relación con los demás en la conversación, a través de la cual se construye el orden social. Pero el acto de posicionarse no solo se limita a la relación con los demás, sino a todo lo que hace parte del contexto sociocultural en el que el acto dialógico tiene lugar. Así, una persona puede tomar una posición frente a cualquier objeto que haga parte del contexto sociocultural del que hace parte. Asimismo, para que haya una toma de posición, debe haber una evaluación de ese objeto por parte del hablante. Una vez que el hablante ha evaluado el objeto y se ha posicionado frente a él, puede alinearse o no con su interlocutor.

De manera dinámica, evaluación, posicionamiento y (des-) alineamientos están integrados en la noción de stance, sobre todo en la manera como ha sido desarrollada por Du Bois (2007, 2002), Jaffe (2009) y Kiesling (2009). Stance es la adopción de una postura social que incluye la evaluación de un elemento existente en el contexto discursivo (stance focus) por parte de un actor social (stancetaker), el posicionamiento con respecto a este elemento y el alineamiento o des-alineamiento entre los diferentes actores sociales que participan en la interacción (Ver Figura 1).

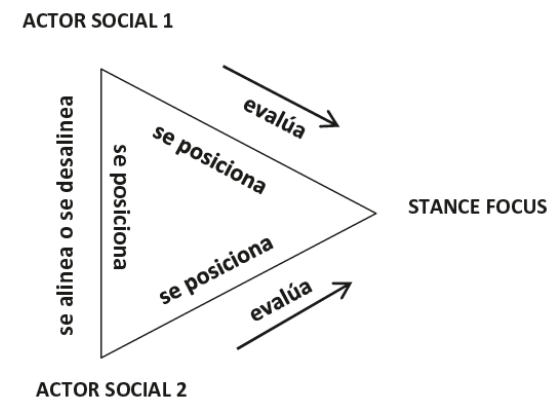

Figura 1. El triángulo de stance. Adaptado de Du Bois (2007) y Kiesling (2011).

Adoptar una postura social o stance es un acto singular y unificado que tiene lugar en la interacción social. Un hablante puede expresar una o varias posturas al mismo tiempo o incluso moverse de una postura o posición a otra (footing). ${ }^{7}$ Los hablantes, a veces, adoptan posturas sociales, aunque no necesariamente, a través del uso de formas lingüísticas particulares que pueden ser indexicales (Blommaert 2007; Silverstein 2003), es decir que tienen un lazo natural con lo que socioculturalmente puede ser una lengua, pero que pueden indexar también algunas posturas de poder, de exclusión, de jerarquía, pero también de afiliación a un grupo social, etc.

\section{1 "a mi cuando me dicen indioh...", posturas de afiliación}

El siguiente extracto del corpus de la ciudad de Cali $(2)^{8}$ ilustra la forma como algunos hablantes quichuas ecuatorianos negocian sus identidades sociales adoptando posturas de afiliación para mitigar las relaciones de poder y de jerarquía entre ellos y la población mayoritaria hispanohablante. Se trata de un extracto de conversación en el que participamos Anita y yo, el investigador. Anita es una mujer quichua de unos cuarenta años, nacida en Cali, que reivindica

\footnotetext{
${ }^{7}$ Ver Goffman (1981: 128) para la definición del concepto de footing o el hecho de moverse dinámicamente de una postura a otra para marcar una diferencia, una similitud, una afiliación, etc., es decir, construir significado social.

${ }^{8}$ Corpus de primera mano recolectado etnográficamente en el centro de la ciudad de Cali en 2011.
} 
À paraître dans Contacto y migración. Desafíos metodológicos en la sociolingüística hispánica actual. Special issue, Iberoromania, De Gruyter, 2020.

su pertenencia a la comunidad quichua tanto en su forma de vestir como en el uso del quichua y del español andino. Por su parte, yo, me posiciono como hablante del grupo mayoritario, aun cuando he desarrollado relaciones de confianza con Anita durante el tiempo que he pasado con ella en la ciudad de Cali.

Le pregunto a Anita si ha tenido problemas con la gente de Cali por pertenecer a la comunidad de los quichuas ecuatorianos. Anita responde que no, pero le insisto (línea 3). A esta insistencia, Anita responde que hay personas que la tratan despectivamente de "india" (línea 4).

(2)

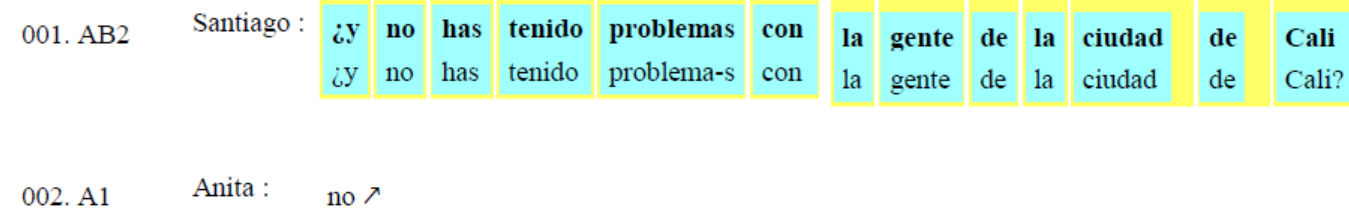

\begin{tabular}{|l|l|l|l|l|}
\hline yo & cuando & me & dicen \\
yo & dicendo & me & indios ['indion] $\nearrow$ \\
\hline le & digo & gringos $\searrow$ \\
le & digo & gringos $\searrow$ \\
\hline
\end{tabular}

Resulta interesante las formas lingüísticas escogidas por Anita, dentro de su repertorio lingüístico, para expresar lo difíciles que pueden ser, por momentos, las relaciones con la gente de Cali. La codificación del corpus nos muestra que la mayor parte de los elementos lingüísticos utilizados en este contexto puede ser atribuida a cualquier variedad del español, de manera indistinta (anotados en dos niveles). Y, por momentos, la hablante utiliza formas más particulares, marcadas (líneas 4 y 6), que pueden ser atribuidas a la manera de hablar en Cali, es decir, al español caleño. Esta asociación se hace gracias a que observamos en la mayoría de las formas lingüísticas marcadas (transcritas en una sola línea) la aspiración del fonema /s/. Este fenómeno de variación fonética es una particularidad ya descrita en la literatura sobre el español de Cali (Brown \& Brown 2012; File-Muriel \& Brown, 2010) y que puede tener una función social asociada a la identidad caleña. También, por momentos, es re-utilizada por los quichuas de Cali para afiliarse socialmente al grupo mayoritario (Sánchez Moreano 2018). 
À paraître dans Contacto y migración. Desafíos metodológicos en la sociolingüística hispánica actual. Special issue, Iberoromania, De Gruyter, 2020.

Anita parece posicionarse, a través del uso de formas lingüísticas en las que se observa el debilitamiento del fonema /s/, como alguien capaz de hablar como la gente local. Al mismo tiempo, Anita se posiciona como alguien que intenta negociar su condición de indígena en la ciudad y minimizar así la asimetría que hay en las relaciones de poder entre grupos mayoritarios y minoritarios en la ciudad de Cali. Para hacerlo, Anita adopta la forma de hablar de la gente de Cali indexada en esta particularidad fonética. En otras palabras, Anita adopta una voz tipificada en términos de Agha (2005), representada en la aspiración del fonema /s/, que indexa la forma de hablar de un grupo en particular, la de los caleños.

\section{2. "Vous voulez combien?", des-alineamiento y exclusión}

De la misma manera, en el siguiente ejemplo (3), observamos cómo la utilización del francés, lengua oficial en la Guayana francesa, sirve también para adoptar una postura social en la interacción. Se trata de un extracto de conversación entre Lorenzo y una clienta brasilera en el puesto de venta de legumbres de Lorenzo en el mercado de Cayena. Luego de ver que la clienta retiró de su canasta algunos pimientos que Lorenzo ya había pesado (algo que a él le disgusta ya que le hace perder tiempo con otros clientes), este último se enfada y le retira la canasta diciéndole en la línea 020 "pasa, pasa" (devuélveme para volver a pesarlos, en realidad). La clienta intenta dar una explicación diciendo que los ha sacado porque no estaban muy verdes (línea 021). Lorenzo se los devuelve, después de haberlos pesado una vez más y al hacerlo, utiliza formas lingüísticas que pueden ser asociadas tanto al español como al portugués brasilero, pero que la clienta comprende muy bien porque, no solo la réplica está acompañada de la acción, sino que también ambos comparten recursos lingüísticos comunes asociados socioculturalmente al portugués brasilero y al español.

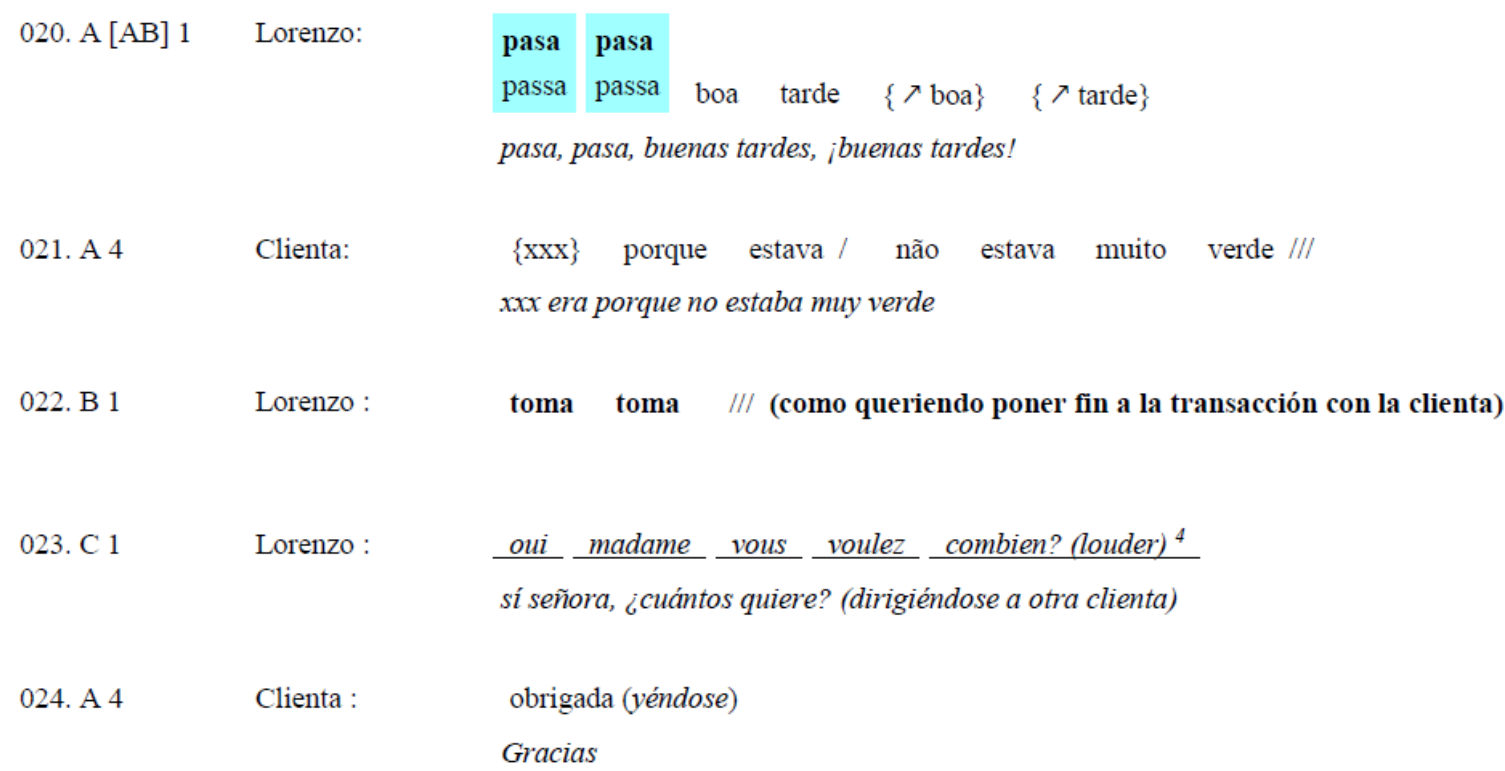

Sin embargo, lo que resulta interesante es la utilización de formas lingüísticas del francés, por parte de Lorenzo en la línea 023, para, por un lado, atender a otra clienta y, por otro, dar por terminada la interacción con la clienta brasilera. Dicha replica se realiza con un poco más de intensidad prosódica y, además de terminar la interacción precedente, sirve como instrumento de posicionamiento para excluir a la hablante brasilera del marco de la conversación.

Sería fácil afirmar que Lorenzo utiliza el francés como una herramienta de poder para afirmarse como el patrón de su puesto de legumbres en el mercado o para marcar una frontera o una diferencia entre él y la clienta brasilera, o incluso para expresar una jerarquía gracias al prestigio 
À paraître dans Contacto y migración. Desafíos metodológicos en la sociolingüística hispánica actual. Special issue, Iberoromania, De Gruyter, 2020.

que puede tener el francés en este contexto. Todas estas pueden ser hipótesis viables. Sin embargo, nada, en la mera conversación nos permite afirmarlo, es por esto que la observación etnográfica y el conocimiento del contexto sociocultural pueden contribuir a la realización de análisis más pertinentes, pero sobre todo, el hecho de que este tipo de posturas sociales son adoptadas por los hablantes de manera recurrente y en diversos contextos.

Lo que sí podemos afirmar con certeza es que Lorenzo asume una postura social frente a la clienta y crea una relación de poder que puede dar indicio de procesos de construcción identitaria más amplios y ligados al uso de diferentes recursos lingüísticos. De hecho, la adopción de este tipo de posturas por parte de Lorenzo son recurrentes como se ha observado durante el trabajo de observación etnográfico.

\section{3. "Ojo con loh chuguah", afiliación efímera de hablantes del grupo mayoritario}

Finalmente, el ejemplo siguiente $(4)^{9}$ muestra cómo los hablantes de los grupos mayoritarios eligen formas lingüísticas particulares, disponibles en sus repertorios lingüísticos, para expresar afiliaciones efímeras en la interacción. En este extracto, también de la ciudad de Cali, Manuela, una mujer quichua de unos 60 años que trabaja en el centro y declara hablar quichua y español andino, está atendiendo su puesto de venta al tiempo que departe con varios de sus colegas o vecinos de puesto. Estos colegas pueden ser indígenas, afrocolombianos, caleños sin pertenencia étnica, etc. Su vecino de enfrente, quien manifiesta justamente no tener afiliación étnica y se considera originario de Cali, le pide a Manuela que le cuide su puesto de ventas mientras él va a hacer unas fotocopias muy cerca de allí (001).

El hablante utiliza inequívocamente formas del español caleño. Pero, de manera interesante, la respuesta que obtiene por parte de Manuela es una afirmación en quichua (línea 002.) El colega reconoce este elemento como quichua y le replica a Manuela introduciendo otra forma lingüística asociada al quichua o al español andino, "shuhua" en el turno número 003.

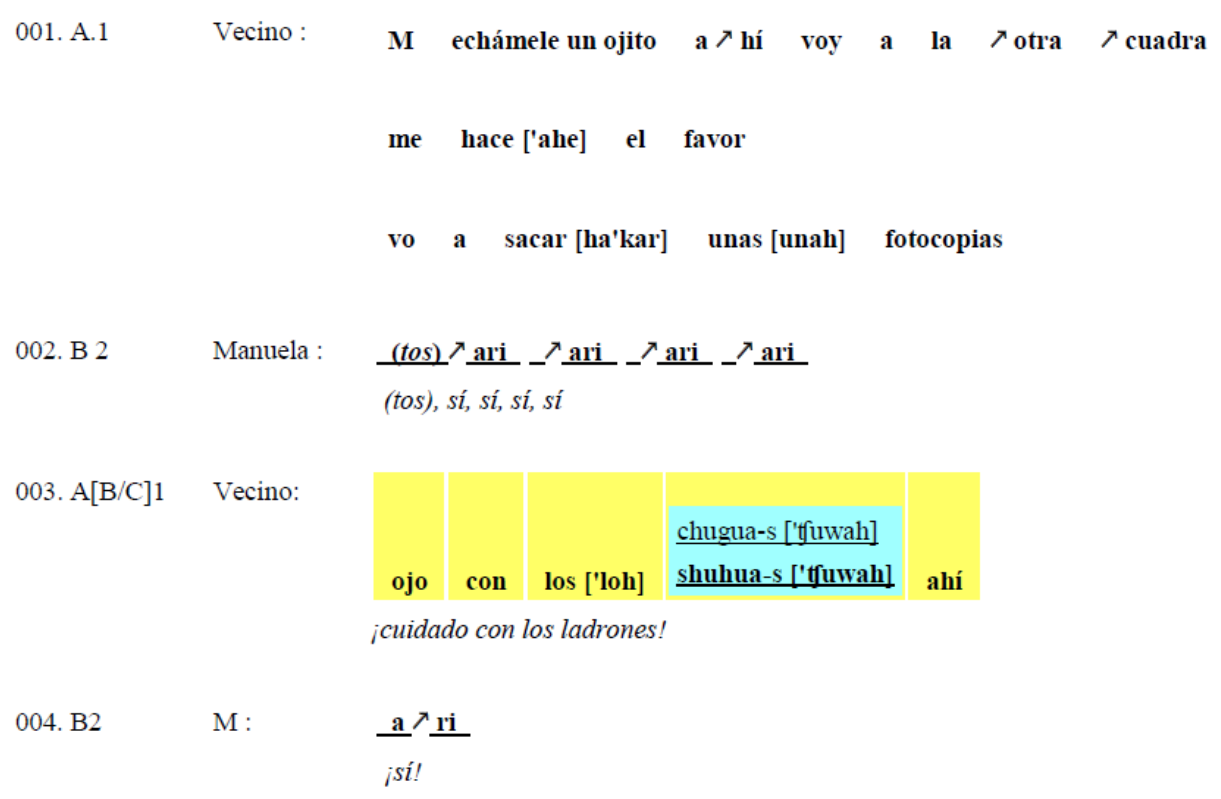
${ }^{9}$ Ejemplo extraído de Léglise y Sánchez Moreano (2017), recolectado también etnográficamente en el centro de
Cali en 2015. 
À paraître dans Contacto y migración. Desafíos metodológicos en la sociolingüística hispánica actual. Special issue, Iberoromania, De Gruyter, 2020.

En términos de la codificación secuencial (Auer 1995), observamos en la secuencia B2 A[B/C]1 que el hablante 1 (Vecino) selecciona una forma lingüística en particular que puede ser interpretada como B o C (quichua o español andino), dos variedades de lengua habladas por la hablante 2 (Manuela). Al hacerlo, el hablante 1 muestra que es capaz de comprender y utilizar algunas formas del repertorio lingüístico de la hablante 2 (Manuela) sin él ser necesariamente hablante de quichua. Esto nos hace pensar en la noción de language crossing (Rampton 2005) para explicar cómo los hablantes que no hacen parte de un grupo étnico negocian su aceptación en dicho grupo a través del uso de formas lingüísticas propias de ese grupo.

Aquí el hablante utiliza la forma "shuhua" con fines específicos: asumir una posición o postura de afiliación efímera con respecto a Manuela como estrategia para convencerla de que cuide su puesto mientras él está ausente. En este sentido, esta postura puede interpretarse como una manera de afiliarse brevemente al grupo de los quichuas buscando establecer lazos de solidaridad en sus prácticas laborales, es decir, colaboración puntual para cuidar un puesto mientras se está ausente. También, se puede interpretar como una estrategia para referirse a los posibles shuhuas (ladrones) sin que estos se enteren de que se está hablando de ellos. Una vez más, la etnografía nos permite afirmar que este tipo de prácticas es recurrente en el centro de Cali en donde permanentemente se tejen relaciones de solidaridad entre colegas vendedores. Lo curioso es que, normalmente, este tipo de estrategias es recurrente entre miembros del mismo grupo, pero se puede observar también en hablantes externos al grupo, como en el caso del vecino de Manuela.

\section{Conclusiones y perspectivas}

Este artículo tenía por objetivo mostrar cómo los individuos, en contextos de movilidad transnacional y de superdiversidad, son susceptibles de adoptar posturas sociales a través del uso de formas lingüísticas socioculturalmente asociadas a las diferentes lenguas (pero también a variedades diatópicas o estilísticas, registros, voces estereotipadas, etc.) que han adquirido a lo largo de sus trayectorias de vida y que son parte de sus repertorios lingüísticos (Busch 2012, 2017). Se propuso que, para la comprensión de dichos usos y adopción de posturas, el análisis conversacional y de la interacción debía estar fundamentado en la observación etnográfica de prácticas de lenguaje socialmente situadas. También se propuso como herramienta analítica, la noción de stance (Du Bois 2007; Kiesling 2009; Jaffe 2009) que nos permite comprender cómo los hablantes asumen posturas sociales de afiliación o exclusión en la interacción social.

La combinación del análisis conversacional y de la interacción, de la observación etnográfica y de la adopción de posturas sociales (stance), puede contribuir a hacer más fino el análisis de las prácticas de lenguaje de los hablantes y del uso socialmente significativo que hacen de sus recursos linguiísticos heterogéneos. Siguiendo Léglise (2013), consideramos que el análisis secuencial de la conversación propuesto por Auer (1995) constituye una base importante susceptible de ser adaptada a corpus heterogéneos (plurilingües, pluridialectales, pluriestilísticos, pluriacentuales) como los que fueron recolectados en Cali y en Cayena. Pero dicha adaptación debe tener en cuenta, no el nivel de los códigos en contacto, sino el nivel de los recursos lingüísticos, en la terminología de Blommaert y Backus (2011) o de las linguistic features en la terminología de Jorgensen et al. (2011).

Por su parte, la observación etnográfica de las interacciones en las que están imbricadas las conversaciones es fundamental, como lo sugieren Bucholtz y Hall (2005). La perspectiva etnográfica permite análisis más finos, sin tomar el riesgo de tomar atajos y relacionar rápidamente las formas lingüísticas con el mundo social y la construcción de identidad. En el caso de los contextos evocados en este artículo, la observación etnográfica nos permite afirmar, por ejemplo, que lo que hacen peruanos y brasileros con sus recursos lingüísticos en Cayena es una práctica social recurrente en las que no se negocia necesariamente una afiliación a un Estado 
À paraître dans Contacto y migración. Desafíos metodológicos en la sociolingüística hispánica actual. Special issue, Iberoromania, De Gruyter, 2020.

o a otro, sino que se expresa lo heterogéneo que son las trayectorias diversificadas y transnacionales de los hablantes. De la misma manera, la observación etnográfica nos permite observar, por ejemplo, que el uso de ciertos recursos lingüísticos, tanto en Cayena como en Cali, contribuye a la adopción de posturas sociales de afiliación, a la negociación de dichas posturas, y eventualmente, a la construcción de identidades locales y globales.

Por supuesto, estos análisis podrían ser completados con otros métodos, también de tipo posestructuralistas, como el análisis de retratos lingüísticos (Busch 2017, 2018) para describir y comprender las trayectorias multimodales y la experiencia vivida del lenguaje de los hablantes en situaciones de movilidad transnacional, transterritorial y de superdiversidad. O, también, podrían ser completados por el análisis de escalas sociolingüísticas y cronotopos (Blommaert 2015) para comprender la complejidad de los lazos que unen las formas lingüísticas con el mundo social en el sentido en que los fenómenos sociales obedecen a escalas de jerarquización en los que cada una tiene un valor socialmente significativo.

\section{Referencias}

Agha, Asif (2005): „Voice, Footing, Enregisterment“, Journal of Linguistic Anthropology 15 (1), pp. 38-59.

Auer, Peter (2005): „A postscript: Code-switching and social identity“, Journal of Pragmatics 37, pp. 403-410.

(1995): „The pragmatics of code-switching: A sequential approach“, en: Milroy, Lesley; Muysken, Pieter (eds.): One speaker, two languages: Cross-Disciplinary Perspectives on Code-Switching, Cambridge: Cambridge University Press, pp. 115-135.

Blommaert, Jan (2015): „Chronotopes, Scales, and Complexity in the Study of Language in Society“, Annual Review of Anthropology 44 (1), pp. 105-116.

- (2007): „Sociolinguistics and discourse Analysis: Orders of indexicality and Polycentricity“, Journal of Multicultural Discourses 1 (2), pp. 115-130.

Blommaert, Jan; Backus, Ad (2011): „Repertoires revisited: 'Knowing language' in superdiversity“, Working Papers in Urban Language \& Literacies 67, pp. 1-26.

Blommaert, Jan; Rampton, Ben (2011): „Language and Superdiversity“, Diversities 13 (2), pp. $1-21$.

Boutet, Josiane; Fiala, Pierre; Simonin-Grumbach, Jenny (1976): „Sociolinguistique ou sociologie du langage ?", Critique 344, pp. 68-85.

Brown, Earl K.; Brown, Esther (2012): „Syllabe-final and syllabe-initial /s/ reduction in Cali, Colombia: One variable or two?“", en Richard, File-Muriel; Rafael, Orozco (eds.): Colombian varieties of Spanish, Madrid/Frankfurt: Iberoamericana/Vervuert, pp 89106.

Bucholtz, Mary (1999): „You da man: Narrating the racial other in the production of white masculinity“, Journal of Sociolinguistics 3 (4), pp. 443-460.

Bucholtz, Mary; Hall, Kira (2008): „Finding identity: Theory and data“, Multilingua - Journal of Cross-Cultural and Interlanguage Communication 27, pp. 151-163.

Busch, Brigitta (2018): „The language portrait in multilingualism research: Theoretical and methodological considerations“, Working Papers in Urban Language \& Literacies 236, pp. 1-13.

- (2017): „Expanding the Notion of the Linguistic Repertoire: On the Concept of Spracherleben-The Lived Experience of Language“, Applied Linguistics 38 (3), pp. 340-358. [en línea: https://doi.org/10.1093/applin/amv030, 01/08/2019]

- (2012): „,The Linguistic Repertoire Revisited“, Applied Linguistics 33 (5), pp. 503-523.

Davies, Bronwyn; Harré, Rom (1990): „Positioning: The Discursive Production of Selves“, JTSB Journal for the Theory of Social Behaviour 20(1), pp. 43-63. 
À paraître dans Contacto y migración. Desafíos metodológicos en la sociolingüística hispánica actual. Special issue, Iberoromania, De Gruyter, 2020.

Du Bois, John W. (2007): „The stance triangle“, en: Englebretson, Robert (ed.): Stancetaking in Discourse, subjectivity, evaluation, interaction, Amsterdam / Philadelphia: John Benjamins Publishing Company, pp. 139-182.

- (2002): „Stance and Consequence“, The Annual Meeting of the American Anthropological Association, New Orleans.

File-Muriel, Richard; Brown, Earl. K. (2010): „The gradient nature of s-lenition in Caleño Spanish“, University of Pennsylvania Working Papers in Linguistics 16 (2), pp. 46-55.

García, Ofelia; Flores, Nelson; Spotti, Massimiliano (2016): „Introduction Language and Society. A Critical Poststructuralist Perspective“, en: García, Ofelia; Flores, Nelson; Spotti, Massimiliano (eds.): Bilingualism, Multilingualism, Globalization, and Superdiversity, Oxford: Oxford University Press, pp. 1-16.

Goffman, Erving (1981): „Footing“, en: Forms of talk, Philadelphia: University of Pennsylvania Press, pp. 127-179.

Gumperz, John J. (1964): „Linguistic and social interaction in two communities“, American Anthropologist 66 (6), pp. 137-153.

Heller, Monica (2007): „Bilingualism as ideology and practice“, en: Heller, Monica (ed.): Bilingualism a social approach, London: Palgrave McMillan, pp. 1-22.

Hymes, Dell. (1974): Foundations in Sociolinguistics: An ethnographic approach, Philadelphia: University of Pennsylvania Press.

Jaffe, Alexandra M. (2009): Sociolinguistic perspectives on stance. Oxford: Oxford University Press.

Jørgensen, Jens Normann; Karrebaek, Martha Sif; Madsen, Lian Malai; Møller, Janus Spindler (2011): „Polylanguaging in Superdiversity“, Diversities 2 (13), pp. 22-37.

Kiesling, Scott Fabious (2011): „Stance in context: Affect, alignment and investment in the analysis of stancetaking", The iMean Conference, Bristol: The University of the West of England.

- (2009): "Style as a Stance: Stance as the Explanation for Patterns of Sociolinguistic Variation", en: Jaffe, Alexandra (ed.): Stance: Sociolinguistic Perspectives, Oxford: Oxford University Press, pp. 171-194.

- $\quad$ (2004): „Dude“, American Speech 79 (3), pp. 281-305.

Kroskrity, Paul V. (2006): „Language Ideologies“, en: Duranti, Alessandro (ed.): A Companion to Linguistic Anthropology, Oxford: Blackwell, pp. 496-517.

Léglise, Isabelle (2018): „Pratiques langagières plurilingues et frontières de langues“, en: Auzanneau, Michelle; Greco, Luca (eds.): Dessiner les frontières, Paris: ENS Editions, pp. 143-169.

(2013): Multilinguisme, variation, contact. Des pratiques langagières sur le terrain à l'analyse de corpus hétérogènes (HDR Dissertation, Institut National des Langues et Civilisations Orientales). [en línea: http://hal.archives-ouvertes.fr/tel-00880500/ 01/08/2019]

Léglise, Isabelle; Sánchez Moreano, Santiago (2017): „From varieties in contact to the selection of linguistic resources in multilingual settings", en: Bassiouney, Reem (ed.): Identity and Dialect Performance, ). New York: Routledge Taylor and Francis, pp. 141-159.

Makoni, Sinfree; Pennycook, Alastair (2005): „Disinventing and (Re)Constituting Languages“, Critical Inquiry in Language Studies 2 (3), pp. 137-156. [en línea: https://doi.org/10.1207/s15427595cils0203_1, 01/08/2019].

Meissner, Fran; Vertovec, Steven (2015): „Comparing super-diversity“, Ethnic and Racial Studies 38 (4), pp. 541-555. [en línea: https://doi.org/10.1080/01419870.2015.980295, 01/08/2019].

Mendoza-Denton, Norma (2014): Homegirls language and cultural practice among latina youth gangs, London: Wiley-Blackwell. 
À paraître dans Contacto y migración. Desafíos metodológicos en la sociolingüística hispánica actual. Special issue, Iberoromania, De Gruyter, 2020.

Meyerhoff, Miriam (2009): „Men argue, but the Women duz Trace: Gender and language variation in Bequia“, Sargasso 2008-09, pp. 115-132.

Migge, Bettina; Léglise, Isabelle (2013): Exploring language in a multilingual context: Variation, Interaction and Ideology in language documentation, Cambridge: Cambridge University Press.

Motta González, Nancy; Posso, Jeanny (2007): Hacia el reconocimiento de una identidad indígena urbana en Cali. Estudio etnológico de las comunidades indígenas ubicadas en el municipio de Santiago de Cali, Kofán, Guambiana, Quichua, Inga, Yanacona, Nasa, Cali: Alcaldía Santiago de Cali/Ministerio del Interior y de Justicia y Fundación General de Apoyo.

Myers-Scotton, Carol (2001): „The matrix language frame model. Developments and responses“, en: Jacobson, Rodolfo (ed.): Codeswitching Worldwide II, Berlin: Mouton de Gruyter, pp. 23-58.

Ochs, Elinor (1992): „Indexing Gender“, en: Duranti, Alessandro; Goodwin, Charles (eds.): Rethinking Context : Language as an Interactive Phenomenon, Cambridge: Cambridge University Press, pp. 335-358.

Otheguy, Ricardo; García, Ofelia; Reid, Wallis (2015): „Clarifying translanguaging and deconstructing named languages: A perspective from linguistics“, Applied Linguistics Review 6 (3), pp. 281-307. [en línea: https://doi.org/10.1515/applirev-2015-0014, 01/07/2019]

Rampton, Ben (2005): Crossing: Language and Ethnicity among Adolescents (2 ${ }^{\mathrm{a}}$ ed.) Manchester / Northampton MA: St. Jerome Publishing.

Sacks, Harvey; Schegloff, Emanuel A.; Jefferson, Gail (1974): „A simplest systematics for the organization of turn-taking for conversation“, Language 50, pp. 696-735.

Sánchez Moreano, Santiago (2018): „Negociación de posicionamientos sociales (stances) a través del debilitamiento del fonema /s/ en el español hablado por los quichuas ecuatorianos en Cali (Colombia)“, Pragmática Sociocultural / Sociocultural Pragmatics 6 (1), pp. 33-70. [en línea: https://doi.org/10.1515/soprag-2017-0019, 01/07/2019]

- (2017): „Enoncés de type OV et positionnements sociaux dans l'espagnol parlé par les Quichuas équatoriens à Cali, Colombie“. Sociolinguistics Studies. Equinox 11 (1), 65-105. [en línea: https://doi.org/10.1558/sols.30850, 01/07/2019]

Silverstein, Michael (2003): „Indexical order and the dialectics of sociolinguistic life“, Language \& Communication 23 (3-4), pp. 193-229. [en línea: https://doi.org/10.1016/S0271-5309(03)00013-2, 01/07/2019]

Spotti, Massimiliano; Blommaert, Jan (2017): „Bilingualism, Multilingualism, Globalization, and Superdiversity: Toward Sociolinguistic Repertoires“, en García, Ofelia;" Flores, Nelson; Spotti, Massimiliano (eds.): The Oxford Handbook of Language and Society, Oxford: Oxford University Press, pp. 161-178.

Vertovec, Steven. (2009): Transnationalism, London/New York: Routledge.

- (2007a): „New directions in the Anthropology of Migration and Multiculturalism“, Ethnic and Racial Studies 30 (6), pp. 961-978. [en línea: https://doi.org/10.1080/01419870701599416, 01/07/2019]

- (2007b): „Super-diversity and its implications“, Ethnic and Racial Studies 30 (6), pp. 1024-1054.

\section{Convenciones de transcripción}

/ pausa 1 segundo 
À paraître dans Contacto y migración. Desafíos metodológicos en la sociolingüística hispánica actual. Special issue, Iberoromania, De Gruyter, 2020.

// pausa 2 segundos

/// pausa 3 segundos

$\nearrow$ entonación ascendente

$\searrow$ entonación descendente

::: alargamiento de vocal

subrayado itálica: francés

subrayado negrita: quichua

negritas : espagnol

letra normal (standard): español no marcado

[transcripción fonética]

\{\} : solapamiento

$\mathrm{xxx}$ : inaudible

(indicación para-verbal: risas, gritos, cantos, etc.) 